\title{
Causas públicas, historias privadas: los derechos reproductivos y el aborto en Bolivia
}

Causes publiques, histoires privées : les droits reproductifs et l'avortement en Bolivie

Public Causes, Private Stories: Reproductive Rights and Abortion in Bolivia

Virginie Rozée, Susanna Rance y Silvia Salinas Mulder

\section{(2) OpenEdition}

Journals

Edición electrónica

URL: http://journals.openedition.org/bifea/8067

DOI: $10.4000 /$ bifea. 8067

ISSN: 2076-5827

Editor

Institut Français d'Études Andines

Edición impresa

Fecha de publicación: 1 diciembre 2016

Paginación: 389-406

ISSN: 0303-7495

Referencia electrónica

Virginie Rozée, Susanna Rance y Silvia Salinas Mulder, «Causas públicas, historias privadas: los derechos reproductivos y el aborto en Bolivia », Bulletin de l'Institut français d'études andines [En línea], 45 (3) | 2016, Publicado el 08 diciembre 2016, consultado el 05 noviembre 2020. URL : http:// journals.openedition.org/bifea/8067 ; DOI : https://doi.org/10.4000/bifea.8067

\section{(c) (i) $\ominus$}

Les contenus du Bulletin de l'Institut français d'études andines sont mis à disposition selon les termes de la licence Creative Commons Attribution - Pas d'Utilisation Commerciale - Pas de Modification 4.0 International. 


\title{
Causas públicas, historias privadas: los derechos reproductivos y el aborto en Bolivia
}

\author{
Virginie Rozée* \\ Susanna Rance ${ }^{* *}$ \\ Silvia Salinas Mulder***
}

\begin{abstract}
Resumen
El artículo reflexiona en torno a elementos que afectan la decisión de las mujeres acerca de la reproducción y del aborto. Contribuye a los debates sobre políticas, campañas activistas y el Código Penal boliviano, en un contexto en el que las desigualdades y la violencia basada en el género ponen desafíos a la eficacia de las tecnologías y las leyes para garantizar el ejercicio del derecho a decidir. Las autoras analizan experiencias narradas por las mujeres en dos investigaciones en La Paz y El Alto, y consideran interpretaciones de la participación de los hombres y enfoques desde las masculinidades. El artículo examina diversas situaciones ligadas al aborto que ilustran la complejidad para las mujeres de decidir sobre las cuestiones reproductivas, solas o con la participación de la pareja y de otras personas.
\end{abstract}

Palabras clave: derechos reproductivos, aborto, género, Bolivia, autonomía, masculinidades

\section{Causes publiques, histoires privées : les droits reproductifs et l'avortement en Bolivie}

\section{Résumé}

L'article propose des réflexions sur les éléments qui affectent la décision des femmes concernant leur reproduction et l'avortement. II contribue aux débats autour des politiques, des campagnes militantes

* Socióloga. Institut National d'Études Démographiques (INED), 133 bd Davout, 75980 París, cedex 20, Francia. Email: virginie.rozee@ined.fr

** Socióloga. Institute for Health and Human Development, University of East London, Stratford Campus, Water Lane, London E15 4LZ, Reino Unido. Email: s.rance@uel.ac.uk

${ }^{* * *}$ Antropóloga. Consultora e investigadora independiente: Achumani Bajo, calle 1 Nr. 100, La Paz, Bolivia. Email: ssalinasmu@hotmail.es 
et du Code Pénal bolivien, dans un contexte où les inégalités et la violence basée sur le genre défient l'efficacité des technologies et des lois qui permettent le libre droit de décider. Les auteures analysent les expériences racontées par des femmes dans deux études réalisées à La Paz et à El Alto, et considèrent différentes interprétations de la participation des hommes et approches du champ des masculinités. L'article analyse diverses situations liées à l'avortement qui illustrent la complexité de décider sur les questions reproductives, seules ou avec la participation du partenaire et d'autres personnes.

Mots-clés : droits reproductifs, avortement, genre, Bolivie, autonomie, masculinités

\title{
Public Causes, Private Stories: Reproductive Rights and Abortion in Bolivia
}

\begin{abstract}
This paper offers reflections about elements that affect women's decisions about reproduction and abortion. It contributes to debates about policies, activist campaigns and the Bolivian Penal Code, in a context where inequalities and gender-based violence challenge the efficacy of technologies and laws to guarantee women's ability to exercise the right to choose. The authors analyze women's narratives about their experiences in two studies carried out in La Paz and El Alto, and consider different interpretations of men's participation and approaches from the field of masculinities. The paper examines diverse situations relating to abortion that illustrate the complexity faced by women deciding on reproductive matters, alone or with the participation of partners and others.
\end{abstract}

Keywords: reproductive rights, abortion, gender, Bolivia, autonomy, masculinities

Bolivia figura entre los países de América Latina que tienen un marco político medianamente progresista en materia de derechos reproductivos, expresado por el Estado desde la suscripción, en 1994, del Programa de Acción de la Conferencia Internacional de Población y Desarrollo (CIPD) de El Cairo1. En la Constitución Política del Estado aprobada en 2009, el artículo 66 «garantiza a las mujeres y a los hombres el ejercicio de sus derechos sexuales y sus derechos reproductivos» (Ministerio de la Presidencia, 2009). El aborto inducido está penado de acuerdo al artículo 266 del Código Penal, pero la causal «incesto, violación o daño a la salud de la mujer» es invocada para permitir el «aborto impune»; a partir de una Sentencia Constitucional Plurinacional (2014), esto puede hacerse sin necesidad de apelar a un juzgado. La tendencia hacia la despenalización se traduce también en la oferta institucionalizada de una atención posaborto regulada por sucesivos programas nacionales de salud reproductiva. Dicha atención incluye el tratamiento

1 La definición de los derechos reproductivos asumida por el Estado boliviano desde 1994 se basa en el párrafo 7.3 del Programa de Acción de la CIPD que se refiere al «derecho básico de todas las parejas e individuos a decidir libre y responsablemente el número de hijos, el espaciamiento de los nacimientos y el intervalo entre éstos y a disponer de la información y de los medios para ello y el derecho a alcanzar el nivel más elevado de salud sexual y reproductiva. También incluye su derecho a adoptar decisiones relativas a la reproducción sin sufrir discriminación, coacciones ni violencia, de conformidad con lo establecido en los documento de derechos humanos» (Naciones Unidas, 1994). 
de las «hemorragias de la primera mitad del embarazo» mediante la Aspiración Manual Endouterina (AMEU), el tratamiento farmacológico y el legrado uterino instrumental (Ministerio de Salud y Deportes, 2009).

Los avances en la materia durante las últimas décadas fueron logrados por la confluencia entre distintas fuerzas y actoras/es. Se destacan las campañas hechas por organizaciones de mujeres y consorcios nacionales e internacionales, incluyendo iniciativas de grupos de hombres trabajando sobre género y derechos desde enfoques de masculinidades (aunque el tema del aborto como tal no haya sido priorizado por ellos). También fueron influyentes las acciones individuales de mujeres quienes, en Bolivia como en otros contextos, se apropiaron del Misoprostol para interrumpir embarazos (Arilha \& Barbosa, 1993). Más recientemente, la emergencia del virus del Zika y las microcefalias asociadas al mismo han provocado nuevos debates en la región sobre el impacto en la salud pública $-y$ en los derechos de las mujeres - de las restricciones legales a la práctica del aborto (Román, 2016). Por otro lado, observamos un contexto en el que si bien el activismo en torno al aborto se revitaliza, a diferencia de la década de los años 1990, este ha dejado de ser tema de interés investigativo; así, a pesar de que se realizan algunos estudios, el abordaje es más desde el activismo que desde la academia.

Las mujeres activistas, investigadoras y otras que vivimos los cambios de aquellas décadas podríamos contar historias que revelan dimensiones subyacentes a las versiones públicas sobre el ejercicio de los derechos reproductivos en Bolivia. Algunas vivimos nuestros propios abortos, practicados en condiciones menos o más seguras, con decisiones más o menos autónomas; otras no quisimos o no pudimos abortar. Las autoras de este artículo, como tantas mujeres, tenemos lazos de sangre, vida y muerte con los temas de sexualidad, reproducción, fertilidad e infertilidad, pérdida de embarazos, embarazos y abortos deseados y no deseados, partos y nacimientos. Ofrecemos el análisis que sigue para contribuir al debate sobre el Código Penal boliviano y los cambios en las leyes sobre el aborto en la región. Compartimos nuestras reflexiones en torno a algunos elementos que afectan la decisión autónoma de las mujeres sobre la reproducción y, específicamente, en torno al aborto.

Los debates sobre derechos y legislación tienen mucho que ver con las relaciones de género y el poder. Observamos algunos avances en la despenalización del aborto en Bolivia. A la vez, en un contexto donde persisten las desigualdades y la violencia basada en el género, cuestionamos la eficacia de las tecnologías e inclusive de las leyes para garantizar el ejercicio del derecho a decidir. ¿Dónde queda la autonomía de las mujeres en sus relaciones con los hombres y otras personas que influyen en sus decisiones? ¿Será que los marcos emancipatorios corren el riesgo de convertirse en nuevos marcos regulatorios (De Souza Santos, 2001), y que la «libertad de decidir» puede entenderse como equivalente a «decidir abortar»? Para indagar sobre estas cuestiones, a partir de una revisión de las políticas, las campañas y los datos demográficos, analizamos las experiencias y percepciones de las mujeres relatadas en algunas investigaciones cualitativas y reflexionamos sobre el rol de los hombres en las decisiones y prácticas del aborto. 


\section{EL ABORTO EN CONTEXTO: AVANCES Y RESTRICCIONES}

En los avances políticos de las últimas décadas respecto al tema del aborto, fueron influyentes la recuperación de la democracia en Bolivia (1982) y la apertura del debate sobre mortalidad materna, salud reproductiva y planificación familiar como algo distinto a la imposición del control natal. Este espacio de debate fue impulsado por diferentes instancias y coaliciones: las Conferencias Internacionales de Población y Desarrollo de las Naciones Unidas, especialmente la de El Cairo (1994); movilizaciones de organizaciones de mujeres y otras, como la Campaña 28 de Septiembre por la Despenalización del Aborto en América Latina y el Caribe, realizada anualmente desde 1993 para llamar la atención pública sobre los efectos del aborto inseguro en la salud y la vida de las mujeres; y acciones coordinadas desde 2015 bajo el paraguas del Pacto Nacional por la Despenalización del Aborto. El Pacto lleva a cabo la campaña «Despenaliza mi decisión», que propone eliminar la sanción contra mujeres que voluntariamente interrumpen un embarazo, y realizar una estrategia de prevención de la violencia sexual (Pacto Nacional por la despenalización del aborto, 2016). Las movilizaciones lograron impactos en las políticas, en programas específicos de salud sexual y reproductiva y en proyectos de ley sobre los derechos reproductivos.

No obstante estos avances, los indicadores de salud reproductiva de las mujeres han cambiado relativamente poco en las últimas décadas. Aunque los métodos anticonceptivos son ahora más conocidos y utilizados, persiste una proporción significativa de embarazos no deseados y una importante incidencia del aborto sobre la mortalidad materna. Bolivia tiene la tercera tasa más alta de mortalidad materna en la región latinoamericana después de Haití y Guyana: de acuerdo al Estudio Nacional de Mortalidad Materna (Ministerio de Salud, 2016), la razón de mortalidad materna (RMM) para el año 2011 fue de 160 por 100000 nacidos vivos, comparada con una RMM de 187 obtenida para el año 2000. En 2011, el $68 \%$ de las muertes maternas ocurrieron en mujeres indígenas, y el $14 \%$ correspondió a adolescentes entre 14 y 19 años (Ministerio de Salud, 2016).

Las principales causas directas de muerte materna identificadas en 2011 continuaron siendo las mismas que las que fueron registradas en el año 2000: hemorragias (59\%), hipertensión (19\%) y aborto (13\%). Respecto a la magnitud de la práctica del aborto en Bolivia, solo existen estimaciones extrapoladas a partir de otros datos. El trasfondo de sanción, castigo y estigmatización que ha rodeado el tema dificulta su estimación. Una investigación realizada por IPAS pronosticóque «al finalizar el año 2011, aproximadamente 67000 abortos serán practicados y a consecuencia de los mismos, 100 mujeres perderán la vida» (IPAS, 2011: 8).

Los casos de aborto informados a Fiscalía para habilitar su práctica legal siguen siendo pocos, pero su número aumenta cada año. El aborto está tipificado en el Código Penal que establece sanciones de dos a seis años de privación de libertad para quien practica el aborto con o sin el consentimiento de la mujer, y para la propia mujer que decide voluntariamente realizarse este procedimiento. De acuerdo a otra investigación realizada por IPAS (2013), entre 2008 y 2012 se 
presentaron 775 denuncias por el delito de aborto en las ciudades de La Paz y Santa Cruz, la mayoría contra mujeres acusadas de haber interrumpido un embarazo. Solo 61 de estas denuncias se encontraban en proceso. Las demás fueron desestimadas porque la parte denunciante (por lo general el Ministerio Público o un proveedor de servicios de salud) no hizo un seguimiento, o porque la policía o el sistema judicial no tomaron acciones. Solo un caso terminó con sentencia: el de una mujer guaraya de 28 años quien permaneció 9 meses en la cárcel por haber interrumpido su embarazo. A los riesgos legales se añaden otros, de revictimización y maltrato si la mujer acude a un hospital con complicaciones (Mercado, 2011); y del entramado institucional que obstaculiza la atención a víctimas de delitos sexuales, cuando una mujer que sufrió violación busca un aborto legal (Brañez \& Equipos departamentales, 2015).

Los obstáculos al ejercicio de los derechos reproductivos, y concretamente a la autonomía de las mujeres en sus decisiones sobre el aborto, pueden ser analizados en el marco de diferentes fuerzas cuyos efectos se combinaron en casos específicos. En el campo de la cooperación internacional, la Regla de Obstrucción Global del Congreso de los Estados Unidos (iniciada con la Política de la Ciudad de México en 1984 y reforzada por la Enmienda Smith en 2001) restringió la discusión y las acciones relacionadas con el aborto por parte de organizaciones no gubernamentales (ONG) que recibían fondos de población del gobierno estadounidense. La llamada «Ley Mordaza» influyó en las políticas del gobierno boliviano, que en estas épocas recibía financiamiento para programas de salud reproductiva de la Agencia de los Estados Unidos para el Desarrollo Internacional (USAID, según sus siglas en inglés).

A través de la Conferencia Episcopal, la Iglesia Católica fue otro actor que ejerció influencias para restringir la oferta estatal de servicios anticonceptivos y obstaculizar la liberalización de las leyes sobre el aborto. También hubieron presiones sobre las mujeres desde el campo biomédico y una situación generalizada de inequidad, discriminación y violencia basada en el género. Mostraremos a continuación cómo la operación de estas fuerzas — teorizadas como dominaciones (Rozée, 2007; 2009a; 2009b) o como relaciones de poder permeadas por desigualdades de género (Sawicki, 1991) — puede ser leída en las narrativas de mujeres bolivianas sobre sus decisiones y prácticas ligadas al aborto.

\section{HISTORIAS PRIVADAS DEL ABORTO: ENTRE DECIDIR Y NO DECIDIR}

Las discusiones sobre políticas, campañas e indicadores parten de una perspectiva global sobre el ejercicio de los derechos reproductivos y las prácticas del aborto en Bolivia. Las experiencias narradas en contextos particulares que hemos reunido en nuestras propias investigaciones dan otras visiones de la problemática. Con este análisis, buscamos ampliar la comprensión de situaciones ligadas al aborto que pueden enfrentar las mujeres en sus relaciones con los hombres. 


\section{1. Relatos de las mujeres sobre el papel de los hombres}

Un estudio en tres hospitales públicos de La Paz y El Alto (2004-2005) destacó las representaciones de las mujeres sobre las cuestiones reproductivas y su manejo (Rozée, 2008). El estudio incorporó observaciones dentro de los servicios ginecológicos y obstétricos, entrevistas con 91 mujeres en consulta u hospitalizadas en estos servicios, y talleres sobre salud reproductiva organizados con mujeres de centros de madres y juntas vecinales.

Los discursos de las mujeres estaban atravesados por referencias a normas de género y a la dominación masculina, particularmente cuando hablaron de quién tenía que decidir sobre las cuestiones reproductivas. Las mujeres declararon que los hombres hacían presión sobre ellas para tener relaciones sexuales sin tener en cuenta el periodo menstrual, a veces con violencia y después de haber consumido alcohol. El método del calendario (ritmo) estaba así destinado al fracaso. En un taller con una decena de mujeres de una asociación en La Paz en 2004, ellas declararon que los hombres no las entendían, que imponían sus necesidades (sexuales), y que si un hombre respetaba la decisión de su pareja de no tener relaciones sexuales era porque tenía otra mujer.

Los hombres fueron representados en los relatos de estas mujeres como reacios al uso de métodos anticonceptivos modernos, con el argumento de que con su uso perdían el control sobre la conducta sexual de su pareja e incluso sobre su paternidad. Según ellas, los hombres consideraban estos métodos como propicios para la infidelidad de las mujeres. Una mujer declaró que su esposo estaba en contra de que ella usara estos métodos, diciendo: «Eres mía».

En este contexto, algunas mujeres declararon ocultar a sus esposos que usaban métodos modernos. Otras, por el contrario, dijeron que preferían consultar con su pareja:

Entonces, eso también le voy hablar con él para decidir... Si tal vez la T de cobre o para preguntarle...

Yo he pensado... Ligamento de trompa... para no tener más bebés... Yo, la verdad, no quiero más bebés. Pero, tengo que consultar a mi esposo, qué es lo que dice.

Depende también cual método se puede usar (...). Voy a ver... cuál soporto más y cuál vamos a hacer. Planificación... Depende de mi esposo también, no...

En los hospitales donde se hizo el estudio, para algunos métodos como la anticoncepción quirúrgica voluntaria (AQV) o el dispositivo intrauterino (DIU), se requería el consentimiento y la firma del esposo (algo fuera de la norma establecida), reafirmando así la responsabilidad social de facto y el poder de los hombres relativo a las cuestiones reproductivas dentro de la pareja y la familia. Algunas mujeres preferían entonces descartar esta opción para no convocar a su pareja por miedo a su reacción. Así, se observaron mujeres nuevamente embarazadas sin haberlo querido. 
Los compromisos internacionales y las políticas públicas reconocen la corresponsabilidad de hombres y mujeres en los asuntos de la reproducción; las organizaciones de mujeres defienden el derecho de las mujeres a decidir sobre su propio cuerpo. Sin embargo, según los relatos de las mujeres entrevistadas, ellas consideran que los hombres tienen un papel clave en las decisiones reproductivas y sexuales, lo que se traduce frecuentemente en que son ellos los que deciden solos. Al mismo tiempo, ellas tienen que asumir en la práctica estas decisiones reproductivas y manejar solas las consecuencias. Las normas de género que asignan a las mujeres la responsabilidad del trabajo reproductivo resultan interiorizadas por las mismas mujeres y reforzadas, según ellas, por los hombres. Este poder masculino sobre la reproducción, real o supuesto por las mujeres, limita la autonomía de las mujeres sobre sus cuerpos y puede afectar su salud, en particular en situaciones de embarazo no deseado y aborto.

Un análisis basado en teorías del patriarcado puede ser usado para culpar a los hombres como inhibidores de los derechos reproductivos de las mujeres. Desde una perspectiva de género también se tienen que considerar variantes de las masculinidades y la complejidad de roles y relaciones en diversos contextos y situaciones. Sin embargo, los estudios sobre el aborto en Latinoamérica, como en otras regiones, suelen enfocarse en las mujeres, ocultando la experiencia de «la otra mitad significativa» (Lerner \& Guillaume, 2008: 30) que son los hombres.

\section{2. Dilemas y tensiones sobre el papel de los hombres}

En los años 1990 emergieron en la región latinoamericana distintos movimientos y organizaciones que apostaban por la transformación de las normas, roles y relaciones de género a partir de un trabajo con y desde los hombres orientado hacia la deconstrucción de la masculinidad hegemónica y la creación de nuevas formas y posibilidades de «ser hombres». En ese marco, el Grupo de Trabajo en Masculinidades de Bolivia2 creó un eslogan que afirmaba: «Hablar de género es también hablar de hombres». A la par y de manera creciente, la cooperación internacional, en alguna medida cuestionada por los modestos resultados alcanzados a partir del trabajo con mujeres, decidió apoyar el trabajo con hombres y en masculinidades; pero, frecuentemente sin mucha claridad política y estratégica sobre las implicaciones del trabajo con hombres en relación a la igualdad de género y la autonomía de las mujeres (Tellería Huayllas, 2011).

Se desarrollaron desde 2004 cinco encuentros sobre masculinidades en Bolivia, organizados por la ONG CISTAC (Centro de Investigación Social, Tecnología Apropiada y Capacitación) y el Grupo de Trabajo en Masculinidades, que contribuyeron a la generación e intercambio de conocimientos, así como a la reflexión sobre el papel de los hombres y dimensiones de las masculinidades desde una mirada relacional de género. De una revisión de las memorias de 
dichos encuentros se puede concluir que el embarazo no deseado y el aborto no han sido un tema central en estos espacios; solo en el tercer encuentro de 2008 hubo una presentación titulada «El aborto, posturas de hombres y mujeres del parlamento uruguayo» (Gonzales, 2008). Esto no resulta sorprendente, pues desde una revisión regional reciente, Pecheny (2015: 52) concluyó que «las brechas de investigación sobre varones y aborto son, por así decirlo, totales».

Una de las razones a las cuales se atribuye la escasez de estudios sobre hombres y aborto se refiere a la «construcción social tanto del aborto, como de su abordaje político e investigativo»; en otras palabras, «no se sabe bien cuál es ni cuál debería ser el papel de los varones en relación con el aborto, con su experiencia y práctica, en la lucha por sacarlo de la clandestinidad y obtener el reconocimiento del aborto legal, o en la investigación académica» (Pecheny, 2015: 51). Esta afirmación coincide con la opinión de Jimmy Tellería, director de CISTAC (ONG pionera en el trabajo con hombres desde el enfoque de masculinidades), que piensa que incluso una posición masculina de «apoyo tu decisión» puede conllevar contenidos de poder «perversos» que, en última instancia, expresan y reproducen el orden patriarcal. Por otro lado, opinando sobre la lucha de las mujeres activistas por la despenalización, Tellería percibe que en alguna medida su legitimidad se sustenta en una «esencialidad biológica» que reafirma la idea del aborto como un «asunto de mujeres».

Quizás en torno a estas ambigüedades y complejidades, también surgió el hecho de que el acceso al aborto y la libre decisión no sea una agenda prioritaria de los hombres, y que su participación en movilizaciones relacionadas con estas causas haya sido coyuntural y mayoritariamente en el marco de representaciones institucionales. Algunos estudios encontraron diversos tipos de reacción e involucramiento de los hombres en la toma de decisión frente a un embarazo o aborto (Dibbits \& Terrazas, 1995; Viveros Vigoya \& Facundo Navia, 2012; Zamberlin et al., 2012). Así, en un estudio realizado en Bogotá sobre el papel de las masculinidades en el aborto, el desinterés de los hombres por estos temas fue interpretado por algunas mujeres como una confirmación de que

a ellas se les ha impuesto la carga de los hechos de la procreación, mientras a los varones no se les ha inculcado el sentido de responsabilidad con la misma (Viveros Vigoya \& Facundo Navia, 2012: 147).

Según este argumento, la falta de involucramiento de los hombres no sería necesariamente una demostración de respeto a la autonomía de las mujeres y su «libre decisión» frente a un embarazo o aborto. El documento Mitos y realidades. El aborto en Bolivia resalta que la «mujer-madre (...) por lo general está sola cuando debe pasar la experiencia de un aborto, no importa si lo decidió ella o se lo impuso el marido», y enfatiza que «el peso de la decisión de abortar no se distribuye equitativamente» (Alanes, 1995: 20).

En aparente contradicción, otros estudios aluden a un involucramiento activo, incluso dominante, de los hombres en las decisiones de las mujeres respecto al aborto. Los hallazgos son diversos, así como las formas de análisis y representaciones sobre el no/involucramiento de los hombres en situaciones de aborto, que son 
influenciadas por las distintas teorías de poder y los abordajes de los asuntos de género. El reto está en evidenciar esta diversidad, enfocarse en los significados tomando en cuenta relaciones, situaciones y contextos específicos, así como reconocer la complejidad que engloba dilemas, tensiones y contradicciones.

Consecuentemente, similares formas de no/participación pueden tener distintas connotaciones en función del contexto específico, a su vez que los individuos pueden tener diferentes formas de involucramiento en distintas situaciones:

[...] cada experiencia de aborto se inscribe en un contexto social y relacional específico y (...) es tan relevante el contexto social y relacional como las características individuales del sujeto (Viveros \& Facundo, 2012: 150)

En todo caso, la autonomía de las mujeres en las decisiones y prácticas reproductivas recibe la influencia de las normas de género interiorizadas y de las estrategias de adaptación o resistencia. Las decisiones y el acceso al aborto ilustran bien la complejidad de manejar sola o con la pareja las cuestiones reproductivas, en contextos donde

los sujetos que toman la decisión del aborto, mujeres y hombres, son sujetos constituidos por $-\mathrm{y}$ constituyentes de - una organización jerárquica de género (Viveros Vigoya \& Facundo Navia, 2012: 160).

Así, un libro que agrupa las historias de 20 mujeres bolivianas que abortaron encuentra que hay una diversidad de situaciones, pero que en la mayoría de los casos los hombres no asumen responsabilidad relativa a la decisión de abortar o no, y tienden a alejarse de la búsqueda y del proceso de aborto:

A lo largo de la investigación, el aborto aparece como un asunto de mujeres. Los hombres no se involucran en su práctica. Especialmente en el caso de las parejas adolescentes (...). En parejas estables de gente adulta hay mayor tendencia en los hombres a apoyar el proceso, acompañar a la mujer para que se haga la intervención y pagar. Sin embargo, las mujeres sienten que de todos modos son ajenos a la experiencia en sí (Aliaga et al., 2000: 93-94).

En el Manual para el aborto seguro y libre en Bolivia (Cuerpa Autónoma, 2014), los testimonios indican, por un lado, que los hombres no quieren involucrarse en la decisión del aborto; y por el otro, que las mujeres — por miedo o por el sentimiento que tienen que decidir por ellas mismas - no informan a los hombres sobre el embarazo y la decisión de abortar. Al contrario, otro estudio afirma que «los hombres también abortan», analizando que «el hombre utiliza el poder socialmente otorgado que ostenta para presionar a la mujer, ya sea en función a que siga con el embarazo o para que lo interrumpa» (Aliaga \& Machicao, 1995: 29).

La revista Debate sobre Derechos Sexuales (Alanes, 2016) resume un debate público en Bolivia en torno a distintos temas relativos a los derechos sexuales, considerado el paraguas amplio que incorpora los derechos reproductivos. Bajo el título «iQuién debe decidir?», se aborda el tema del aborto desde distintas presentaciones, haciendo hincapié en el contexto más allá de las leyes, la necesidad 
de despenalizar el aborto a nivel social, y de fomentar la responsabilidad de los hombres. Ramiro Claure, quien fue director de Marie Stopes Bolivia, enfatizó que muchos hombres «se hacen a la vista gorda», y afirmó:

Tenemos más del 50\% de la responsabilidad, porque somos violadores a los derechos de las mujeres (Alanes ed., 2016: 19).

Actualmente el CISTAC está desarrollando una teleserie «Hombres» que incluye el tema de aborto, los discursos masculinos de control del cuerpo y toma de decisión, y los efectos del machismo en la vida de las mujeres.

\section{3. ¿QUIÉN DECIDE? HISTORIAS SOBRE PÉRDIDAS E INTERRUPCIONES DEL EMBARAZO}

Un estudio realizado en El Alto en 2009 analizó una serie de historias personales de mujeres en torno a temas de los derechos que incluyeron el aborto. El Alto es una ciudad colindante con La Paz, habitada principalmente por migrantes de áreas rurales y mineras. En una investigación-acción participativa realizada en coordinación con el Centro de Promoción de las Mujeres Gregoria Apaza (Rance, 2009), 46 mujeres y 22 hombres fueron entrevistadas/os individualmente (o en algunos casos en pares) por 8 investigadoras ( 7 mujeres y un hombre). Las personas entrevistadas tenían entre 12 y 48 años. El tema de la entrevista fue libre, con una sola pregunta inicial seguida por mínimas intervenciones posteriores de las investigadoras: ¿Puedes relatar algún hecho en tu vida sexual o reproductiva que te haya impactado especialmente?

Cada investigador/a eligió su propio tema para el análisis y la escritura de un capítulo del libro Abriendo el paquete envuelto: violencias y derechos en la Ciudad de El Alto (Rance coord., 2009). Entre los múltiples temas tocados en las entrevistas, 17 de las 46 mujeres aludieron a un total de 29 abortos o pérdidas del embarazo en sus trayectorias de vida, dentro y fuera de relaciones estables de pareja. Según sus relatos, los abortos fueron realizados en consultorios privados, hospitales o casas particulares, usando métodos tradicionales (infusión de yerbas) y modernos (legrados y fármacos). Las pérdidas fueron asociadas, en sus relatos, con hechos accidentales como caídas, situaciones de violencia como golpes o empujones, así como esfuerzos físicos de la mujer como levantar cosas pesadas. Se analizaron estas historias, buscando entender el significado de cada caso y algún patrón común en el conjunto de las narraciones.

En una primera ronda del análisis, se clasificó cada aborto o pérdida según el grado de agencia o sujeción expresado en el discurso de la mujer respecto a las circunstancias en que ocurrió. Un hallazgo sorprendente fue que solo uno de los 29 abortos fue descrito como algo decidido autónomamente por la mujer en cuestión. En dos casos las mujeres dijeron que deseaban abortar, pero necesitaban lograr un acuerdo con sus parejas para poder hacerlo; y dos mujeres se retrataron como obligadas a tomar la decisión de abortar por las situaciones que vivían, como si ellas no tuviesen otra opción. La singularidad del discurso de la decisión 
autónoma recuerda lo dicho por un médico de área en El Alto en otra investigación, quien describió así el fenómeno escaso del embarazo planificado:

en realidad, es como una aguja en un pajar, son muy pocos (Rance, 1995: 121).

En el estudio de 2009, ¿en qué términos se aludió al proceso de una decisión autónoma, tan cercana al ideal de las campañas por los derechos, y tan lejos de la experiencia narrada por la mayoría de las mujeres entrevistadas?

En [nombre de ciudad], nuevamente quedé embarazada. Esta vez no había mutuo acuerdo. Al director del hospital, le rogué, le imploré. Me apoyó y dijo que fuera al hospital al día siguiente. Mi esposo había ido a La Paz. Yo le había avisado sobre el legrado. No estaba muy de acuerdo pero me dejó (Rance coord., 2009: 55 - Historia de Ana³)

En esta historia ejemplar notarán, sin embargo, que la mujer aludió al apoyo «rogado»al director del hospital y al consentimiento renuente del esposo que la «dejó» practicar el legrado. No obstante los obstáculos que tuvo que superar, esta mujer se representó como actora que decidió imponer su voluntad y negociar la interrupción del embarazo, un legrado instrumental practicado de manera ilegal en un hospital de provincia.

\section{1. Relatos de agencia, ambivalencia, coerción y aceptación}

¿Qué hay de los otros veintiocho abortos y de las otras dieciséis mujeres? Aparte de los cinco casos mencionados arriba, donde las mujeres declararon su deseo de interrumpir o perder el embarazo, hubieron otros con diferentes posiciones narradas de ambivalencia, coerción o aceptación de un proceso «natural». Hubo relatos sobre siete pérdidas espontáneas del embarazo, con alivio reportado por algunas mujeres e inclusive la admisión de haber hecho esfuerzos por estimular la pérdida. También hubo mención de cinco abortos «sin datos» porque la mujer en cuestión —la llamaremos Daniela — dio detalles en su entrevista de solo uno de los seis abortos que tuvo.

Los casos que más llamaron nuestra atención fueron los cuatro descritos como «por decisión de otros», y los nueve —el grupo más grande- con relatos de abortos forzados: cinco con intervenciones caseras o médicas, y cuatro causados por golpes de la pareja (no se aclaró si los golpes fueron hechos a propósito para buscar ese fin). Al parecer, en esta muestra cualitativa — sin pretensión de representatividad, pero a la vez sugerente- el aborto autónomamente decidido fue un caso único y los relatos más numerosos fueron sobre abortos decididos, practicados y causados por otros.

Al interpretar estos hallazgos, es pertinente considerar hasta qué punto el relato común de «me obligó», «yo no sabía», «ellos nomás saben» pudo haber

3 Todos los nombres de mujeres entrevistadas son seudónimos. 
respondido a una convención influenciada por las relaciones de género según las cuales una mujer se absuelve de responsabilidad por un hecho considerado inmoral y criminal, retratándose como víctima de presiones ajenas y como sujeta determinada por la autoridad masculina. En el terreno de la ilegalidad, puede ser más prudente representarse como desconocedora de los hechos y los procedimientos. Los detalles narrados tienen fuerte resonancia y trasmiten el sabor de la «no autonomía»:

Una vez perdí a mi hijo por cargar pesado, mi marido no sabe, estaba de dos meses, me pasó cuando viajé esa noche, sólo sentí como si me estuviera bajando mi menstruación. (...) no llegué esa noche y le dije que estaba con mi amiga (Rance coord., 2009: 58 - Historia de Berta).

Me sacaron afuera ellos [su cuñado — autor del embarazo- y el médico], no sé qué han hablado. (...) Me han hecho tomar una pastilla, no sabía para qué era. El sólo me gritaba — «Callada, me vas a hacer caso», me decía-. (...) Yo me asusté tanto que lo único que hice es llorar (Rance coord., 2009: 128 - Historia de Carolina).

\section{2. Una mujer, seis abortos: entre la obligación externa y la decisión autónoma}

Las variaciones en la trayectoria de vida narrada por una mujer, Daniela, ilustran la evolución de la no/autonomía en la interrupción de sucesivos embarazos. Cuando se presentaron públicamente los hallazgos de la investigación con presencia de algunas de las mujeres entrevistadas, Daniela se acercó a su entrevistadora diciendo que no estaba de acuerdo porque faltaban los datos de 5 de sus 6 abortos. Aceptó hacer una nueva entrevista y proporcionó detalles sobre cada uno. Daniela representó la decisión como tomada por otros en los primeros 2 casos (cuando tenía 14 y 22 años), decidida por ella pero bajo presión de las circunstancias en el tercer y cuarto caso (ambos con 26 años), y decidida por ella de manera autónoma en el quinto y sexto caso cuando tenía 32 y 36 años:

1. Fue decidido por otros: mi pareja y mi tía.

2. Mi amiga me obligó. Me dijo, «No vas a destrozar tu vida. iVamos!» Me llevó a la clínica, diciendo «Yo voy a firmar».

3. Yo he decidido. Fue una decisión propia. Ya tenía mi hija — no podía embarazarme nuevamente-.

4. iFue al mes siguiente! Yo he tenido que tomar la decisión. Pensé que no ovulaba -tenía mala información-.

5. No he querido avisar a nadie. He tomado sola la decisión. Fue lo más terrible, con consecuencias. iEn una clínica bien cara!

6. Me he embarazado... yo no quería. Fue decisión mía, pese a que había riesgo para cualquier intervención quirúrgica. (...) Me dio un paro cardiorrespiratorio. Me han reanimado. Fue el último legrado que he hecho. Nunca más volví (Rance coord., 2009: 60) 
Los nuevos datos proporcionados por Daniela hicieron variar la categorización de los veintinueve abortos. En el nuevo esquema, el caso único del aborto autónomamente decidido se convirtió en tres, y dos de las diecisiete entrevistadas aludieron a decisiones autónomas. La evolución en la trayectoria de Daniela fue la expresión de etapas de su vida en que ella se sintió con menor o mayor capacidad para decidir y actuar por su cuenta. En ciertos otros momentos, ella pudo reconocerse —en aquel tiempo o reflexionando años después_ como agente con «decisión propia», tomando «sola la decisión»; o declarando: «fue decisión mía» (aun habiéndose sentido obligada por circunstancias fuera de su control).

\section{3. Mitigar la culpa del hecho}

En sus relatos, se evidencia la dificultad expresada por las mujeres entrevistadas de declararse como decisoras sobre su vida sexual y reproductiva. En varias de las historias, el protagonismo fue atribuido a hombres apoyándose mutuamente (por ejemplo la pareja y un médico), o a mujeres con mayor asertividad o autoridad (la tía o la amiga). La investigación cualitativa indaga sobre sentires, discursos, representaciones y no hechos (dejamos la noción de la objetividad y conductas observadas a otros paradigmas de investigación). No nos compete establecer si hubo o no hubo embarazo obligado, aborto forzado, decisión tomada a espaldas de la mujer en cuestión. Lo que sí podemos registrar es que en los relatos de estas diecisiete mujeres — habiendo elegido ellas mismas hablar sobre el tema del aborto, entre tantos otros hechos posibles en su vida sexual y reproductivalas representaciones del saber y hacer autónomo en el aborto fueron escasas. El patrón más común fue atenuar, discursivamente, el estigma y el sentimiento de culpa ligado al aborto: quería pero no sabía, fue pero no conocía, hizo o tuvo que decidir, pero fue algo obligado (Rance coord., 2009: 130).

Para profundizar la reflexión sobre nuestros hallazgos, recurrimos a algunos textos que nos parecían especialmente relevantes por su enfoque en experiencias de las mujeres y toma de decisión sobre el aborto en diferentes contextos: el estudio de Whittaker (2002) en área rural de Tailandia, el análisis de Lafaurie et al. (2005) de las perspectivas de las mujeres sobre el aborto medicamentoso en cuatro países latinoamericanos y la revisión de la literatura sobre experiencias con el aborto medicamentoso en países donde hay restricción legal por Zamberlin et al. (2012). Estos estudios muestran cómo la decisión de abortar o no, y las experiencias del aborto, también reciben la influencia del lugar de residencia de la mujer (país y zona urbana o rural); la situación de vida (como grado de pobreza, migración); y el acceso a determinados métodos para abortar, en particular con la expansión del uso del aborto medicamentoso. El estudio de Zamberlin et al. nota que las mujeres de menores ingresos no tienen un abanico de métodos seguros para elegir, pero reporta que ellas perciben que usar medicamentos es menos riesgoso que introducir objetos o usar otros métodos tradicionales. Encuentra que «las mujeres toman decisiones pragmáticas sobre el método abortivo basadas en sus posibilidades que no necesariamente constituyen una "elección real"». 
También comenta que «las parejas hombres involucrados en la decisión de abortar usualmente tienen un rol activo en obtener el medicamento, particularmente en contribuir económicamente y buscar de dónde comprarlo» (Zamberlin et al., 2012: 6).

Llama la atención que en estos trabajos sobre las experiencias de las mujeres, la toma de decisión sobre el aborto en diferentes contextos y el uso del aborto medicamentoso (Whittaker, 2002; Lafaurie et al., 2005; Zamberlin et al., 2012), hay mención sobre el rol de las parejas y familiares, así como situaciones de violencia, pero no se menciona el aborto forzado. ¿En qué momentos la «ayuda» de la pareja llega a ser coerción? Si el aborto medicamentoso evita los riesgos de otros métodos, ¿se estimará más prudente eludir el análisis de casos donde se usó de manera forzada o sin consentimiento de la mujer? Cuestionamos la construcción de asociaciones implícitas entre la presencia de la pareja o familiar y la «ayuda», y entre el uso de una tecnología segura y una decisión autónoma por parte de la mujer. En el estudio de El Alto, varias de las mujeres entrevistadas mencionaron a parejas y parientes que tuvieron incidencia directa sobre la interrupción del embarazo, a veces contra su voluntad. No buscamos argumentar a favor de un método por encima de otros, sino develar las tensiones de género y poder en los relatos personales de embarazos y abortos, que rara vez aluden al derecho a decidir de las mujeres.

\section{CONCLUSIÓN: ESCENAS Y ESCENARIOS DEL ABORTO DETRÁS DEL TELÓN}

La ligazón entre el aborto y el derecho a decidir está fuertemente instaurada en las campañas feministas desde hace varias décadas. El eslogan lleva implícita la noción de una mujer que decide por sí misma y actúa en defensa de su propio sentido de lo que desea, lo que le conviene, lo que su cuerpo exige, lo que ella estima mejor para su salud y su vida. Ante un embarazo no esperado, forzado, no deseado, no viable y/o difícil de asumir, se supone que una mujer —en términos idealespuede tomar la decisión de abortar. El rol de las leyes y la justicia, la sociedad y los servicios de salud, sería — nuevamente en términos ideales — respaldar a la mujer y facilitar su toma de decisión sin presiones y con una información adecuada. Si ella decide abortar, en términos ideales contaría con el acceso a los medios, la tecnología, un espacio y un apoyo — profesional, si lo requiere- para que ella pueda interrumpir el embarazo en condiciones seguras.

Este modelo ideal del derecho a decidir contrasta con los hechos reportados de enorme inequidad en el acceso que tienen las mujeres a la información, el respaldo, las tecnologías y la libertad de actuar sin la amenaza de denuncias, extorsión, juicios o encarcelamiento. Cuestiona, asimismo, la sobrevaloración que se hace de la información como factor clave de autonomía reproductiva:

la capacidad de absorción, el procesamiento, uso y capacidad de transmitir información pasan por filtros individuales y colectivos que 
reproducen los patrones sociales y culturales, a su vez condicionados por las múltiples relaciones de poder: de género, generacionales y étnicas. Consecuentemente, las personas necesitan más que información para tomar y promover decisiones informadas (Salinas Mulder, 2001: 326).

Es en contextos específicos, mediados por la cultura y las normas sociales, además de la religión y las leyes, donde se puede evaluar el grado de autonomía y acceso a la toma de decisión y a la práctica correspondiente, por parte de mujeres en momentos particulares de su vida. Las narrativas públicas de las campañas, muchas veces sustentadas en argumentos de salud y supervivencia y enfocadas en cambios legales y de políticas, rara vez aluden a las historias personales que cuentan las mujeres de los pormenores — días, horas, gestos, silencios, viajes, golpes, dolores, alivios - de abortos o embarazos perdidos, procedimientos hechos con o sin su voluntad o consentimiento.

Detrás del telón se juegan escenarios donde las mujeres rara vez se representan como autónomas en cuanto a sus decisiones y prácticas. Dadas las barreras legales imperantes y el agobio de las relaciones de poder mediadas por desigualdades sociales y normas de género, son más comunes las historias femeninas del saber secuestrado, las prácticas obligadas, la suerte puesta en manos de otros/as que tienen el conocimiento y las tecnologías para interrumpir el embarazo. En el contexto boliviano, detrás de un aborto que hacia fuera puede representar o interpretarse como seguro, o fruto del ejercicio del derecho a decidir, se esconden dinámicas y relaciones de poder que ponen en cuestión la medida en que la mujer ejerce un derecho y toma su propia decisión.

En su libro publicado inicialmente en 1984 y reeditado en 2003, Kathleen McDonnell exploró el reto que puede significar para las feministas reconciliar nuestra «política» o «línea oficial» con nuestros sentimientos profundos; y la dificultad de hacer públicas las contradicciones que podemos observar entre la política y la experiencia personal. Señala:

... la experiencia del aborto de las mujeres no está siendo abordada e integrada en la forma en que hablamos políticamente sobre el tema. (...)

Estamos hablando sencillamente de una elección de traer a la discusión pública algunas de las cuestiones que hemos discutido en forma privada desde hace tanto tiempo. (...) Ha habido una necesidad entre las mujeres para este tipo de reevaluación, pero lo que nos ha impedido hacerlo ha sido mayormente el miedo: miedo de a dónde nos puede conducir, miedo de crear divisiones entre nosotras, miedo a que nuestros opositores/as la puedan usar en contra nuestra. (...) Debemos confiar en que (...) llegaremos de nuevo a nuestro punto de inicio — el aborto es un derecho de las mujerespero con una comprensión cambiada y más profunda. (McDonnell, 2003: 23 y 26).

Afirmamos, a partir de nuestro análisis, que el ejercicio de los derechos reproductivos recibe influencias de las condiciones sociales y materiales de vida, la pertenencia étnica, las ideologías imperantes y los sistemas de género. El acceso al aborto seguro — en Bolivia como en muchos otros contextos_ está atravesado 
por las normas de género interiorizadas por hombres y mujeres, con leyes y políticas que las campañas activistas intentan cambiar. Estos sistemas también se traducen en dilemas para las mujeres sobre el rol de los hombres en las decisiones y prácticas del aborto. En un contexto de condicionamiento legal, donde también existen barreras socioeconómicas, culturales e institucionales que dificultan el acceso al aborto seguro, el llamado «mercado del aborto» (Cuerpa Autónoma, 2014) tiene un impacto directo en la salud, la vida y los derechos de las mujeres, incluso con el uso de nuevas tecnologías como el misoprostol. No obstante los avances logrados por acciones de las propias mujeres -individualmente y en campañas públicas - el entramado que hemos descrito aún constriñe su derecho a decidir sobre el aborto en términos simbólicos y prácticos.

\section{Agradecimientos}

Agradecemos los aportes de Malena Morales, Monica Novillo, Jimmy Tellería y Claudia Columba.

\section{Referencias citadas}

ALANES, Z., 1995 - Mitos y Realidades. El aborto en Bolivia, 32 pp.; La Paz: Servicio de Información para el Desarrollo/Population Council.

ALANES, Z., 2016 (ed.) - Debate sobre Derechos sexuales, 36 pp.; La Paz: CISTAC.

ALIAGA BRUCH, S. \& MACHICAO BARBERY, X., 1995 - El aborto: Una cuestión no sólo de mujeres, 123 pp.; La Paz: CIDEM.

ALIAGA BRUCH, S., QUITÓN PRADO, M. \& GISBERT, M. E., 2000 - Veinte Historias, un mismo tema: aborto, 107 pp.; La Paz: Population Council.

ARILHA, M. \& BARBOSA, R. M., 1993 - Cytotec in Brazil: "At Least It Doesn't Kill". Reproductive Health Matters, 2: 41-52.

BRÁÑEZ, P. \& EQUIPOS DEPARTAMENTALES, 2015 - Aplicación de la Sentencia Constitucional 206/2014 en los centros de salud pública. Campaña 28 de septiembre, Bolivia.

CUERPA AUTÓNOMA. POR LA VIDA DE LAS MUJERES, 2014 - Manual para el aborto seguro y libre en Bolivia (https://fr.scribd.com/doc/238178205/Manual-para-elaborto-seguro-y-libre-en-Bolivia)

DE SOUZA SANTOS, B., 2001 - Para um novo senso comum: a ciencia, o direito e a política na transicao paradigmática, s.n.; Sao Paulo: Cortez. 3ed.

DIBBITS, I. \& TERRAZAS, M., 1995 - Hablar sobre el aborto no es fácil, 50 pp.; La Paz: Tahipamu.

GONZÁLES, F., 2008 - Aborto, posturas de hombres y mujeres del parlamento uruguayo. In: 3er encuentro sobre masculinidades. Memoria Bolivia 2008 (J. Tellería Huayllas \& M. Espinoza, eds.): 26-30; La Paz: CISTAC.

IPAS, 2011 - Las cifras hablan. El aborto realizado en condiciones de riesgo es un problema de salud pública, 15 pp.; La Paz: IPAS.

IPAS, 2013 - La criminalización del aborto en Bolivia (infografía); La Paz: IPAS.

LAFAurie, M. M., GROSSMAN, D., TRONCOSO, E., BILlinGS, D. L. \& CHÁVEZ, S., 2005 - Women's Perspectives on Medical Abortion in Mexico, Colombia, Ecuador 
and Peru: A Qualitative Study. Reproductive Health Matters, 13 (26): 75-83 (http:// www.rhm-elsevier.com/article/S0968-8080\%2805\%2926199-2/pdf).

LERNER, S. \& GUILLAUME, A., 2008 - La participación de los varones en la práctica del aborto. La construcción del conocimiento en América Latina. Revista Latinoamericana de Población, 2: 29-45.

MCDONNELL, K., 2003 - Not an easy choice. Re-examining abortion, 157 pp.; Toronto: Second Story Press.

MERCADO, M. L., 2011 - El aborto provocado revela situaciones de extrema violencia. Opinion.com.bo, 11 de diciembre (http://www.opinion.com.bo/opinion/ articulos/2011/1211/noticias.php?id=35007\&calificacion $=5$ )

MINISTERIO DE LA PRESIDENCIA, 2009 - Bolivia. Constitución Política del Estado Plurinacional de Bolivia (http://www.presidencia.gob.bo/documentos/ publicaciones/constitucion.pdf)

MINISTERIO DE SALUD, 2016 - Estudio de Mortalidad Materna 2011 Bolivia. Resumen Ejecutivo; La Paz: Ministerio de Salud.

MINISTERIO DE SALUD Y DEPORTES, 2009 - Manual de Normas, Reglas, Protocolos y Procedimientos Técnicos para el Manejo de las Hemorragias de la Primera Mitad del Embarazo; La Paz: Ministerio de Salud y Deportes.

NACIONES UNIDAS, 1994 - Plan de Acción de El Cairo.

PACTO NACIONAL POR LA DESPENALIZACIÓN DEL ABORTO, 2016 - Propuesta para la Cumbre de Justicia.

PECHENY, M., 2015 - Varones y Aborto: Acompañarlas en lo que ellas decidan: aborto y participación de los hombres. In: Investigación sobre aborto en América Latina y El Caribe: una agenda renovada para informar políticas públicas e incidencia (S. Ramos, ed.): 41-56; Lima: CLACAI.

RANCE, S., 1995 - Aborto, Género y Salud Reproductiva; El Alto, Bolivia: Proyecto de Fortalecimiento de la Atención Primaria en el Distrito III de la Ciudad de El Alto, Misión de Cooperación Técnica Holandesa, CIDEM. Informe inédito.

RANCE, S., 2009 (coord.) - Abriendo el paquete envuelto: violencias y derechos en la Ciudad de El Alto, 135 pp.; El Alto, Bolivia: Centro de Promoción de la Mujer Gregoria Apaza, Solidaridad Internacional Bolivia.

ROMÁN, V., 2016 - El zika despierta debate sobre aborto seguro y legal en América Latina. Scientific American en español, 19 de febrero (https://www.scientificamerican.com/ espanol/noticias/el-zika-despierta-debate-sobre-aborto-seguro-y-legal-en-americalatina/)

ROZÉE, V., 2007 - Les patrons culturels du comportement reproductif et sexuel dans les Andes Boliviennes. Nuevo Mundo, Mundos Nuevos, 7 (http://nuevomundo.revues. org/document3168.html).

ROZÉE, V., 2008 - L'application de la Convention sur l'élimination de toutes les formes de discrimination à l'égard des femmes en Bolivie. Le cas des droits reproductifs et sexuels (La Paz, El Alto). Les Cahiers des Amériques latines, 53: 191-208.

ROZÉE, V., 2009a - La problématique de l'avortement en Bolivie. Recherches Féministes, 22 (2): 77-95.

ROZÉE, V., 2009b - La domination masculine et l'image de la 'Madresposa'. L'exercice des droits reproductifs et sexuels des femmes boliviennes. Les Cahiers du Genre, 46: 177-197.

SALINAS MULDER, S., 2001 - Demography from the soul. In: Cultures of Populations. Population Dynamics and Sustainable Development (S. H. Syed, ed.): 301-361; París: UNESCO. 
SAWICKI, J., 1991 - Disciplining Foucault: Feminism. Power, and the Body, xiii + 130 pp.; Nueva York \& Londres: Routledge.

TELLERÍA HUAYLLAS, J., 2011 - Reflexiones sobre el trabajo en masculinidades, 59 pp.; La Paz: Solidaridad Internacional Bolivia. Serie Hilvanando.

VIVEROS VIGOYA, M. \& FACUNDO NAVIA, A., 2012 - El lugar de las masculinidades en la decisión del aborto. Sexualidad, Salud y Sociedad. Revista Latinoamericana, 12: 135163(http://www.clacaidigital.info:8080/xmlui/bitstream/handle/123456789/462/ SexSaludySoc2012(12)p135_163.pdf?sequence=1\&isAllowed =y)

WHITTAKER, A., 2002 - 'The truth of our day by day lives': Abortion decision making in rural Thailand. Culture, Health \& Sexuality: An International Journal for Researá, Intervention and Care, 4 (1): 1-20 (http://www.tandfonline.com/doi/ abs/10.1080/136910502753389350)

ZAMBERLIN, N., ROMERO, M. \& RAMOS, S., 2012 - Latin American women's experiences with medical abortion in settings where abortion is legally restricted. Reproductive Health, 9 (34): 1-11 (http://www.ncbi.nlm.nih.gov/pmc/articles/PMC3557184/) 